\title{
Sex Differences in Age and Comorbidities for COVID-19 Mortality in Urban New York City
}

\author{
Eyal Klang ${ }^{1} \cdot$ Shelly Soffer ${ }^{2}$ (D) - Girish Nadkarni ${ }^{3} \cdot$ Ben $_{\text {Glicksberg }}{ }^{4} \cdot$ Robert Freeman $^{1} \cdot$ Carol Horowitz $^{5}$. \\ David L Reich ${ }^{1,3} \cdot$ Matthew A Levin ${ }^{1,6}$
}

Accepted: 21 July 2020 / Published online: 9 August 2020

(C) Springer Nature Switzerland AG 2020

\begin{abstract}
Previous studies demonstrated a higher COVID-19 fatality rate in men. The aim of this study was to compare age and comorbidities between women and men who died from COVID-19. We retrospectively analyzed data of COVID-19 patients hospitalized to a large academic hospital system in New York City between March 1 and May 9, 2020. We used a multivariable logistic regression model to identify independently significant variables associated with gender in patients who died from COVID-19. The model was adjusted for age and comorbidities known to be associated with COVID-19 mortality. We identified 6760 patients diagnosed with COVID19. Of these patients, $3018 / 6760$ (44.6\%) were women. The mortality rate was higher for men (women $18.2 \%$ vs. men $20.6 \%, p=$ 0.039 ). Of the patients who died, women were on average 5 years older than men (woman $77.4 \pm 12.7$ vs. men $72.4 \pm 13.0, p<$ 0.001). In the multivariable model, cardiovascular comorbidities were not significantly different between women and men. Chronic kidney disease (aOR for women $0.7,95 \%$ CI $0.5-0.9$ ) and smoking ( $\mathrm{aOR}$ for women $0.7,95 \% \mathrm{CI} 0.5-0.9$ ) were more common in men. Age decile (aOR for women 1.4, 95\% CI 1.3-1.6) and obesity (aOR for women 2.3, 95\% CI 1.8-3.0) were higher in women. This study demonstrates that women who died of COVID-19 showed a similar cardiovascular disease profile as men. Yet, they are 5 years older than men. Investigating the gender impacts of COVID-19 is an important part of understanding the disease behavior.
\end{abstract}

Keywords COVID-19 $\cdot$ Coronavirus $\cdot$ Mortality $\cdot$ Sex $\cdot$ Comorbidities

\section{Introduction}

The coronavirus disease 2019 (COVID-19) is a pandemic viral disease caused by severe acute respiratory syndrome

Eyal Klang and Shelly Soffer contributed equally to this work.

This article is part of the Topical Collection on Covid-19

Shelly Soffer

soffer.shelly@gmail.com

1 Institute for Healthcare Delivery Science, Department of Population Health Science and Policy, Mount Sinai, New York City, NY, USA

2 Faculty of Medicine, Tel Aviv University, Tel Aviv, Israel

3 Department of Medicine, The Hasso Plattner Institute of Digital Health at Mount Sinai; The Charles Bronfman Institute of Personalized Medicine; MSCIC, New York City, NY, USA

4 Department of Genetics and Genomic Sciences, The Hasso Plattner Institute of Digital Health at Mount Sinai, New York City, NY, USA

5 Center for Health Equity and Community Engaged Research, Icahn School of Medicine at Mount Sinai, New York City, NY, USA

6 Department of Anesthesiology, Perioperative and Pain Medicine, Icahn School of Medicine at Mount Sinai, New York City, NY, USA coronavirus 2 (SARS-CoV-2). Several studies have demonstrated that elderly patients with comorbidities are at increased risk of dying from COVID-19. Underlying health conditions such as cardiovascular disease, hypertension, and diabetes mellitus were associated with increased risk of mortality [1-3]. Smoking and obesity were also found to be risk factors $[4,5]$. A number of studies have shown a higher fatality rate in men than in women [6-8]. A research conducted in China reported that $73 \%$ of the mortality cases were men [6]. It was suggested that the gender discrepancy is due to a higher smoking rate in men and subsequently a higher comorbidity rate [9]. The present study was designed to compare age and comorbidities between women and men who died from COVID-19.

\section{Methods}

\section{Study Design}

A multicenter observational retrospective study was conducted using data of COVID-19 patients who presented to a large 
health system in the New York metropolitan area. Data came from five hospital campuses serving different geographic populations (Mount Sinai Hospital, Mount Sinai Brooklyn, Mount Sinai Queens, Mount Sinai Morningside, and Mount Sinai West). The study time frame was between March 1 and May 9, 2020.

The Mount Sinai Institutional Review Board (IRB) approved this study. Informed consent was waived by the IRB committee.

We identified all patients who were positive for COVID-19 by nasopharyngeal swab polymerase chain reaction (PCR) test and were admitted to the hospitals. Demographic data, clinical information, and medical history were retrieved from the hospitals' electronic medical records. Smoking was defined as past or current smoking. Obesity was defined as body mass index (BMI) larger than $30 \mathrm{~kg} / \mathrm{m}^{2}$. For $13.9 \%$ of patients with missing BMI, obesity diagnosis was retrieved from patients' files.

\section{Statistical Analysis}

The analysis was performed with Python (ver. 3.6.5, 64 bits). A $p$ value of $<0.05$ was considered statistically significant.

Age distribution curves were plotted for women and men who died from COVID-19. Gaussian kernel density estimates were fitted to the plots.

Univariate analysis compared age and comorbidities between men and women who died from COVID-19. Categorical variables were compared using chi-square test. Continuous variables were compared using Student's $t$ test.

A multivariable logistic regression model was used to identify independently significant variables associated with gender. The model was adjusted for age decile and comorbidities known to be associated with COVID-19 mortality. Adjusted odds ratios (aOR), 95\% confidence intervals (CI), and $p$ values were calculated for the variables in the models.

\section{Results}

Overall, 6760 patients were diagnosed with COVID-19. Of these patients, 3018/6760 (44.6\%) were women. Of the 6760 patients, $1320(19.5 \%)$ patients died during the study's time frame: $548 / 3018(18.2 \%)$ of the women and $772 / 3742$ $(20.6 \%)$ of the men died $(p=0.039)$.

Of the patients who died, women (mean $77.4 \pm 12.7$ ) were significantly older than men (mean $72.4 \pm 13.0$ ). Figure 1 shows the age distribution curves for women and men who died from COVID-19. The women's age distribution curve is shifted several years forward from the men's age distribution curve.

In univariate analysis (Table 1), no significant gender differences were observed for cardiovascular diseases, diabetes mellitus, and chronic obstructive pulmonary disease. Hypertension (women $79.0 \%$ vs. men $73.8 \%, p=0.035$ ) and obesity (women $37.2 \%$ vs. men $25.5 \%, p<0.001$ ) were significantly more common in women. Chronic kidney disease (women $21.7 \%$ vs. $28.9 \%, p=0.004$ ) and smoking (women $20.4 \%$ vs. men $27.7 \%, p=0.003$ ) were significantly more common in men.

The results of the multivariable model are presented in Table 2. After adjustment, there was still no significant gender difference in cardiovascular comorbidities. Features independently significant in the multivariable analysis were similar to the univariate analysis. Age decile (aOR for women 1.4, 95\%
Fig. 1 Age distribution plot in 1320 patients who died from COVID-19, stratified by sex. The age distribution curve for women is shifted about $5-10$ years ahead of the men's age distribution curve

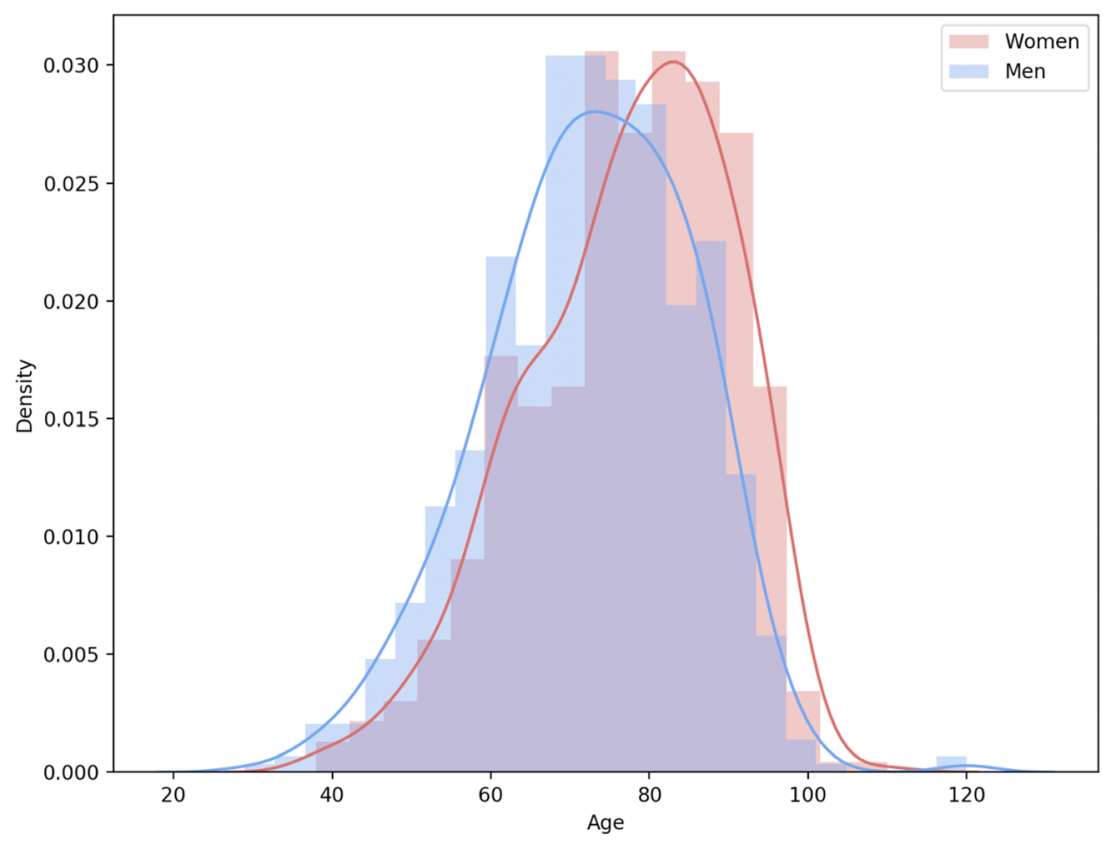


Table 1 Characteristics of the study cohort which included 1320 women and men who died from COVID-19

\begin{tabular}{|c|c|c|c|}
\hline & Women $(n=548,41.5 \%)$ & $\operatorname{Men}(n=772,58.5 \%)$ & $p$ value \\
\hline \multicolumn{4}{|l|}{ Demographics } \\
\hline Age, median (IQR), y & $79.0(68.8-87.0)$ & $73.0(64.0-82.0)$ & $<0.001$ \\
\hline \multicolumn{4}{|l|}{ Race, $N .(\%)$ : } \\
\hline African American & $139(25.4)$ & $163(21.1)$ & 0.081 \\
\hline Caucasian & $149(27.2)$ & $228(29.5)$ & 0.386 \\
\hline \multicolumn{4}{|l|}{ Comorbidities } \\
\hline $\mathrm{CAD}, N .(\%)$ & $160(29.2)$ & $243(31.5)$ & 0.409 \\
\hline $\mathrm{CHF}, N .(\%)$ & 109 (19.9) & $149(19.3)$ & 0.845 \\
\hline $\mathrm{HTN}, N .(\%)$ & $433(79.0)$ & $570(73.8)$ & 0.035 \\
\hline $\mathrm{DM}, N .(\%)$ & $289(52.7)$ & $412(53.4)$ & 0.865 \\
\hline $\mathrm{CKD}, N .(\%)$ & $119(21.7)$ & $223(28.9)$ & 0.004 \\
\hline COPD, $N .(\%)$ & $68(12.4)$ & $97(12.6)$ & 1.000 \\
\hline Cancer, $N .(\%)$ & $89(16.2)$ & $129(16.7)$ & 0.880 \\
\hline Obesity, $N .(\%)$ & $204(37.2)$ & $197(25.5)$ & $<0.001$ \\
\hline BMI, median (IQR) & $28.4(23.5-34.5)$ & $26.9(23.5-30.5)$ & $<0.001$ \\
\hline Smoking, $N .(\%)$ & $112(20.4)$ & $214(27.7)$ & 0.003 \\
\hline
\end{tabular}

$B M I$ body mass index, $C A D$ coronary artery disease, $C H F$ chronic heart failure, $C K D$ chronic kidney disease, $C O P D$ chronic obstructive pulmonary disease, $D M$ diabetes mellitus, HTN hypertension, IQR interquartile range
CI 1.3-1.6) and obesity (aOR for women 2.3 , 95\% CI $1.8-$ 3.0 ) were higher in women. Chronic kidney disease (aOR for women $0.7,95 \%$ CI $0.5-0.9$ ) and smoking (aOR for women $0.7,95 \%$ CI $0.5-0.9)$ were higher in men.

\section{Discussion}

This study demonstrates that in patients who died from COVID-19, no significant difference was noted between

Table 2 Multivariate analysis of age and comorbidities between women and men who died from COVID-19 infection

\begin{tabular}{llrr}
\hline & aOR & $p$ values & $95 \%$ CI \\
\hline Age decile & 1.4 & $<0.001$ & $1.3-1.6$ \\
CAD & 0.8 & 0.183 & $0.6-1.1$ \\
CHF & 1 & 0.810 & $0.8-1.4$ \\
HTN & 1.2 & 0.238 & $0.9-1.6$ \\
DM & 1 & 0.714 & $0.8-1.3$ \\
CKD & 0.7 & 0.015 & $0.5-0.9$ \\
COPD & 1.1 & 0.686 & $0.8-1.5$ \\
Cancer & 1.1 & 0.699 & $0.8-1.5$ \\
Obesity & 2.3 & $<0.001$ & $1.8-3.0$ \\
Smoking & 0.7 & 0.007 & $0.5-0.9$ \\
\hline
\end{tabular}

$a O R$ adjusted odds ratio, $C A D$ coronary artery disease, $C H F$ chronic heart failure, $C I$ confidence interval, $C K D$ chronic kidney disease, $C O P D$ chronic obstructive pulmonary disease, $D M$ diabetes mellitus, $H T N$ hypertension, IQR interquartile range

The model was adjusted for age decile, CAD, DM, CHF, CKD, COPD, obesity, cancer, and smoking cardiac morbidity and gender. Yet, a difference was found for age. Women who died were on average 5 years older than men. Like previous research, our data shows that mortality and hospital admission rates were higher for men.

Previous studies suggested that the gender disparity is a result of behaviors such as smoking and drinking that lead to underlying cardiovascular morbidity [9]. Indeed, in our study, we found that the smoking rate was higher for men. Yet, this difference was not reflected in comorbidity differences between the genders. Obesity was more frequent among women who died.

It is worthy of note that the mortality rate in our cohort is higher than that reported from different locations [10]. Our results are in accordance with other publications from the New York City area, which demonstrated a high COVID-19 mortality rate $[4,11]$.

The link between gender and the severity of COVID-19 disease is still not completely understood. This link can be related to a number of causes including physiological, biological, and sociological factors. In our cohort, there was a fiveyear discrepancy between men and women who died from COVID-19. One possible explanation is that the gender difference results from the association between COVID-19 mortality and underlying health conditions, particularly cardiovascular diseases. In women, cardiovascular disease starts to develop approximately 5-10 years later due to the protective effect of estrogen $[12,13]$. This means that the cardiovascular injury, which may affect the course of COVID-19, begins to accumulate later in women.

Our study is limited to an assessment of gender differences among patients who have died from COVID-19 without 
considering the comorbidity rate of men versus women in the general population. Secondly, this is a retrospective observational study, with all the associated limitations. Thirdly, due to large missing values for race, we did not include this variable in the logistic regression model. Finally, the study represents the urban New York City population, and other populations may have different results.

Investigating the gender impacts of COVID-19 is an important part of understanding the disease behavior. Women who died of COVID-19 showed a similar cardiovascular disease profile as men. Yet, on average, they are 5 years older than men.

\section{Compliance with Ethical Standards}

The Mount Sinai Institutional Review Board (IRB) approved this study. Informed consent was waived by the IRB committee.

Conflict of Interest The authors declare that they have no conflict of interest.

\section{References}

1. Yang J, Zheng Y, Gou X, Pu K, Chen Z, Guo Q, et al. Prevalence of comorbidities in the novel Wuhan coronavirus (COVID-19) infection: a systematic review and meta-analysis. Int J Infect Dis. 2020;94:91-5.

2. Wu C, Chen X, Cai Y, Zhou X, Xu S, Huang H, et al. Risk factors associated with acute respiratory distress syndrome and death in patients with coronavirus disease 2019 pneumonia in Wuhan, China. JAMA Intern Med. 2020;180:934.

3. Zhou F, Yu T, Du R, Fan G, Liu Y, Liu Z, et al. Clinical course and risk factors for mortality of adult inpatients with COVID-19 in
Wuhan, China: a retrospective cohort study. Lancet. 2020;395: 1054-62.

4. Richardson S, Hirsch JS, Narasimhan M, Crawford JM, McGinn T, Davidson KW, et al. Presenting characteristics, comorbidities, and outcomes among 5700 patients hospitalized with COVID-19 in the New York City area. JAMA. 2020;323(20):2052-2059. https://doi. org/10.1001/jama.2020.6775.

5. Klang E, Kassim G, Soffer S, Freeman R, Levin MA, Reich DL. Morbid obesity as an independent risk factor for COVID-19 mortality in hospitalized patients younger than 50. Obesity. 2020;10.1002/oby.22913. https://doi.org/10.1002/oby.22913.

6. Chen T, Wu D, Chen H, Yan W, Yang D, Chen G, et al. Clinical characteristics of 113 deceased patients with coronavirus disease 2019: retrospective study. BMJ. 2020;368:m1091. https://doi.org/ 10.1136/bmj.m1091.

7. Livingston E, Bucher K. Coronavirus disease 2019 (COVID-19) in Italy. JAMA. 2020;323(14):1335.

8. Wenham C, Smith J, Morgan R. COVID-19: the gendered impacts of the outbreak. Lancet. 2020;395(10227):846-8.

9. Lancet $\mathrm{T}$. The gendered dimensions of COVID-19. Lancet. 2020;395(10231):1168.

10. Lq L, Huang T, Wang Y, Wang Z, Liang Y, Tb H, et al. COVID-19 patients' clinical characteristics, discharge rate, and fatality rate of meta-analysis. J Med Virol. 2020;92(6):577-83.

11. Lala A, Johnson KW, Januzzi JL, Russak AJ, Paranjpe I, Richter F, et al. Prevalence and impact of myocardial injury in patients hospitalized with COVID-19 infection. J Am Coll Cardiol. 2020;76(5): 533-546. https://doi.org/10.1016/j.jacc.2020.06.007.

12. Albrektsen G, Heuch I, Løchen M-L, Thelle DS, Wilsgaard T, Njølstad I, et al. Lifelong gender gap in risk of incident myocardial infarction: the Tromsø study. JAMA Intern Med. 2016;176(11): 1673-9.

13. Volgman AS, Bairey Merz CN, Aggarwal NT, Bittner V, Bunch TJ, Gorelick PB, et al. Sex differences in cardiovascular disease and cognitive impairment: another health disparity for women? J Am Heart Assoc. 2019;8(19):e013154.

Publisher's Note Springer Nature remains neutral with regard to jurisdictional claims in published maps and institutional affiliations. 